\title{
Reports, Forum
}

The Person and the Challenges

Volume 3 (2013) Number 2, p. 233-244

\section{Botschaft der polnischen Bischöfe an ihre deutschen Brüder in Christi Hirtenamt}

\section{Hochwürdige Konzilsbrüder!}

Es sei uns gestattet, ehrwürdige Brüder, ehe das Konzil sich verabschiedet, unseren nächsten westlichen Nachbarn die freudige Botschaft mitzuteilen, daß im nächsten Jahr im Jahre des Herrn 1966 die Kirche Christi in Polen und mit ihr zusammen das gesamte polnische Volk das Millenium seiner Taufe und damit auch die Tausendjahrfeier seines nationalen und staatlichen Bestehens begehen wird.

Wir laden Sie hiermit in brüderlicher, aber auch zugleich in feierlichster Weise ein, an den Kirchenfeiern des polnischen Milleniums teilzunehmen; der Höhepunkt des polnischen Te deum laudamus soll Anfang Mai 1966 auf der Jasna Gora, bei der heiligen Mutter Gottes, der Königin Polens, stattfinden.

Die folgenden Ausführungen mögen als historischer und zugleich auch sehr aktueller Kommentar unseres Milleniums dienen und vielleicht auch mit Gottes Hilfe unsere beiden Völker im gegenseitigen Dialog einander noch näherbringen.

Es steht geschichtlich einwandfrei fest, daß im Jahr 966 der polnische Herzog Mieszko I. durch den Einfluß seiner Gemahlin, der tschechischen Königstochter Dombrowka, zusammen mit seinem Hofstaat als erster polnischer Herzog das heilige Sakrament der Taufe empfing.

Von diesem Augenblick an wurde das christliche Missionswirken in Polen seit Generationen durch christliche Apostel schon vorher in unserem Land verkündet im ganzen polnischen Volksraum verbreitet. 
Der Sohn und Nachfolger Mieszkos, Bolesław Chrobry (der Tapfere) setzte das Christianisierungswerk seines Vaters fort und erwirkte vom damaligen Papst Silvester II. die Errichtung einer eigenen polnischen Hierarchie mit der ersten Metropole in Gniezno (Gnesen) und drei Suffraganbistümern Kraków, Wrocław, Kołobrzeg (Krakau, Breslau, Kolberg). Bis 1821 blieb Gniezno weiterhin Metropole des Breslauer Bistums. Im Jahre 1000 begab sich der damalige Herrscher des Römischen Imperiums, Kaiser Otto III., zusammen mit Bolesław Chrobry als Pilger zum Märtyrerschrein des heiligen WojciechAdalbert, der einige Jahre vorher bei den baltischen Preußen den Märtyrertod erlitten hatte. Die beiden Herrscher, der römische und der zukünftige polnische König (er wurde kurz vor seinem Tode zum König gekrönt), gingen barfuß eine weite Strecke Weges zu den heiligen Gebeinen in Gniezno, die sie alsdann mit großer Andacht und innerer Ergriffenheit verehrten.

Das sind die geschichtlichen Anfänge des christlichen Polens und zugleich auch die Anfänge seiner nationalen und staatlichen Einheit. Auf diesen Fundamenten christlich, kirchlich, national und staatlich zugleich wurde sie durch alle Generationen weiter ausgebaut von Herrschern, Königen, Bischöfen und Priestern, 1000 Jahre hindurch. Die Symbiose Christentum, Kirche, Staat bestand in Polen seit Anfang und wurde eigentlich nie gesprengt. Sie erzeugte mit der Zeit die fast allgemeine polnische Denkart: Polnisch ist zugleich katholisch. Aus ihr heraus entstand auch der polnische Religionsstil, in dem seit Anfang an das Religiöse mit dem Nationalen eng verwoben und verwachsen ist, mit allen positiven, aber auch negativen Seiten dieses Problems.

$\mathrm{Zu}$ diesem religiösen Lebensstil gehört auch seit jeher als sein Hauptausdruck der polnische Marienkult. Die ältesten polnischen Kirchen sind der Mutter Gottes geweiht (u.a. die Gnesener MetropolitanKathedrale); das älteste polnische Lied, sozusagen das Wiegenlied des polnischen Volkes, ist ein bis heute noch gesungenes Marienlied: „Bogurodzicadziewica, Bogiem Sławiona Maryja“ (Gottesgebärerin Jungfrau Maria). Die Tradition bringt sein Entstehen mit dem heiligen Wojciech zusammen, ähnlich wie die Legende es mit den polnischen weißen Adlern im Nest von Gniezno tut. Diese und ähnliche Traditionen und Volkslegenden, welche die Geschichtstatsachen wie Efeu umranken, haben das Gemeinsame von Volk und Christentum so eng miteinander verwoben, daß man sie einfach nicht schadlos auseinanderbringen kann. Von ihnen her wird alles spätere polnische Kulturgeschehen, die 
gesamte polnische nationale und kulturelle Entwicklung, bestrahlt, ja sogar zu einem Großteil geprägt.

Die allerneueste Geschichtsschreibung gibt diesen unseren Anfängen folgende politische und kulturelle Bedeutung: „In der Begegnung mit dem Imperium Ottos des Großen vor einem Jahrtausend hat sich Polens Eintritt in die lateinische Christenheit vollzogen, und durch die bewundernswert geschickte Politik Mieszkos I. und sodann Bolesław des Tapferen ist es zu einem gleichberechtigten Glied des universal konzipierten, auf Erfassung der gesamten nichtbyzantinischen Welt gerichteten Imperium Romanum Ottos III. geworden, womit Polen einen entscheidenden Beitrag zu der Gestaltung des östlichen Europa geleistet hat...".

Damit war die Grundlage gelegt und Form und Voraussetzung geschaffen für die kommenden fruchtbaren deutschpolnischen Beziehungen und die Ausbreitung der abendländischen Kultur.

Leider sind die deutschpolnischen Beziehungen im späteren Verlauf der Geschichte nicht immer fruchtbar geblieben und haben sich sozusagen in den letzten Jahrhunderten in eine Art nachbarliche „Erbfeindschaft“" verwandelt. Darüber später.

Der Anschluß des neuen polnischen Königreiches an das Abendland, und zwar mit Hilfe des Papsttums, dem sich die polnischen Könige immer wieder zur Verfügung stellten, brachte im Mittelalter einen in jeder Hinsicht regen und äußerst reichen Austausch zwischen Polen und den abendländischen Völkern, insbesondere mit den süddeutschen Ländern, aber auch Burgund und Flandern, mit Italien und später mit Frankreich und Österreich und den italienischen Renaissancestaaten, wobei natürlicherweise Polen als jüngeres Staatsgebilde, als jüngster von den älteren Brüdern des christlichen Europas, anfangs mehr der nehmende als der gebende Teil war.

Es wurden zwischen Kalisz und Kraków, der polnischen Königsstadt des Mittelalters, und zwischen Bamberg, Speyer, Mainz, Prag, Paris, Köln, Lyon, Clairvaux und Gent nicht nur Waren ausgetauscht. Es kamen aus dem Westen die Benediktiner, die Zisterzienser und später die Bettelorden und erhielten in Polen, im christlichen Neuland, sofort einen schwunghaften Auftrieb; dann kam im Mittelalter das deutsche Magdeburger Recht dazu, das bei polnischen Stadtgründungen große Dienste leistete. Es strömten auch nach Polen deutsche Kaufleute, Architekten, Künstler und Siedler, von denen sehr viele im polnischen Volkstum aufgingen: Ihre deutschen Familiennamen ließ man ihnen. In der großen Krakauer Bürgerkirche St. Maria finden wir noch heute 
die Grabinschriften zahlreicher deutscher Familien aus dem Mittelalter, die mit der Zeit alle polnisch geworden sind, woraus Hitler und andere unseligen Gedenkens den einfachen Schluß zogen, daß Kraków und ganz Polen nur eine deutsche Siedlung seien und demgemäß behandelt werden müssen. Das klassische Beispiel deutschpolnischer Zusammenarbeit in Kultur und Kunst im hohen Mittelalter ist wohl der weltberühmte Bildhauer Veit Stoß aus Nürnberg (Wit Stwosz), der fast sein ganzes Leben lang in Kraków wirkte; seine Werke dort sind alle vom Genius loci der polnischen Umgebung inspiriert: Er schuf in Kraków eine eigene Künstlerschule, die noch Generationen hindurch nachwirkte und das polnische Land befruchtete.

Die Polen haben ihre Brüder aus dem christlichen Westen, die als Boten der wahren Kultur zu ihnen kamen, sehr geehrt und verschwiegen niemals ihre nichtpolnische Stammesherkunft. Wir haben der abendländischen auch der deutschen Kultur wahrhaftig sehr viel zu verdanken.

Aus dem Westen kamen zu uns auch Apostel und Heilige, und sie gehören wohl zu dem Wertvollsten, was uns das Abendland geschenkt hat. Ihr segensreiches soziales Wirken spüren wir vielerorts noch heute. Zu den bekanntesten zählen wir den heiligen Bruno von Querfurt, „Bischof derHeiden“ genannt, der den slawischen und litauischen Nordosten im Einvernehmen mit Bolesław Chrobry evangelisierte. Dann ist ganz besonders zu erwähnen die heilige Hedwig (Jadwiga), Herzogin von Schlesien, aus Andechs gebürtig, Gemahlin des polnischen Piastenfürsten Heinrich des Bärtigen (Brodaty) von Schlesien und Gründerin des Zisterzienserinnenklosters von Trzebnica (Trebnitz), wo sie ihre Grabstätte gefunden hat. Sie ist im 13. Jahrhundert die größte Wohltäterin des polnischen Volkes in den damaligen Westgebieten des Piastenpolens, in Schlesien, geworden. Es steht historisch ziemlich fest, daß sie, um dem polnischen einfachen Volk dienen zu können, sogar die polnische Sprache lernte. Nach ihrem Tode und ihrer baldigen Heiligsprechung strömten ohne Unterlaß Scharen des polnischen und deutschen Volkes zu ihrer Grabstätte in Trzebnica später Trebnitz genannt, und sie tun es noch heute zu Tausenden und Abertausenden. Niemand macht unserer großen Landesheiligen den Vorwurf, daß sie deutschen Geblütes war; im Gegenteil, man sieht sie allgemein von einigen nationalistischen Fanatikern abgesehen als den besten Ausdruck eines christlichen Brückenbaues zwischen Polen und Deutschland an, wobei wir uns freuen, auch auf deutscher Seite recht oft dieselbe Meinung zu hören. Brücken bauen zwischen Völkern können eben am besten nur heilige Menschen, nur solche, die eine lautere Meinung 
und reine Hände besitzen. Sie wollen dem Brudervolke nichts wegnehmen, weder Sprache noch Gebräuche, noch Land, noch materielle Güter; im Gegenteil: Sie bringen ihm höchst wertvolle Kulturgüter, und sie geben ihm gewöhnlich das Wertvollste, was sie besitzen: sich selbst, und werfen damit den Samen ihrer eigenen Persönlichkeit in den fruchtbaren Boden des neuen Missionsnachbarlandes; dieser trägt dann gemäß dem Heilandswort hundertfache Früchte, und zwar Generationen hindurch. So sehen wir in Polen die heilige Hedwig von Schlesien, so sehen wir auch alle anderen Missionare und Märtyrer, die, aus den westlich gelegenen Ländern kommend in Polen wirkten mit dem schon erwähnten Märtyrerapostel Wojciech Adalbert aus Prag an der Spitze. Darin besteht auch wohl der allertiefste Unterschied zwischen echter christlicher Kulturmission und dem sogenannten, heute zu Recht verpönten Kolonialismus.

Nach dem Jahre 1200, als das polnische Land in seinen Menschen und Institutionen immer christlicher wurde, wuchsen ihm eigene polnische Heilige heran.

Schon im 12. Jahrhundert war es der Bischof Stanislaus Szczepanowski von Kraków, Bekenner und Märtyrer, von König Bolesław dem Verwegenen am Altare erschlagen. (Der König selbst starb als heiligmäßiger Büßer in der Verbannung in einem Kloster in Oberösterreich.) Am Grabe des heiligen Stanislaus in der königlichen Domkirche von Kraków entstand das majestätische Lied zu seiner Ehre, heute überall in Polen lateinisch gesungen: «Gaude mater Polonia, prole foecunda nobili...".

Dann erschien am Firmament das heilige polnische Dreigestirn aus der Familie der Odrowaz (ein altes Geschlecht, das lange Jahrhunderte an der Oder in Oberschlesien seinen Sitz hatte). Der größte von ihnen ist der heilige Hyazinth polnisch Jacek genannt, ein Dominikanerapostel, der ganz Osteuropa von Mähren bis zum Baltikum, von Litauen bis Kiew mit Riesenschritten durchmaß. Sein Verwandter, der selige Czesław, ebenfalls Dominikaner, der die damalige Stadt Wrocław gegen die Mongolen verteidigte und im heutigen Wrocław, in der neuerbauten Wojciech (Adalbert) Kirche begraben ist, wird von der frommen Bevölkerung als Patron der aus den Trümmern von 1945 wiedererstandenen Stadt verehrt. Und schließlich ruht in Kraków die selige Bronisława, der Tradition nach Schwester des seligen Czesław, eine Norbertanerin aus Schlesien.

Die Sterne am Heiligenhimmel werden immer mehr: in Sącz die selige Kunigunde, in Gniezno Bogumil und die selige Jolanta, in Masowien 
Władysław, auf der Königsburg in Kraków die heiligmäßige Königin Jadwiga, eine neue polnische Hedwig, die auf ihre Heiligsprechung wartet. Später kamen neue Heilige und Märtyrer dazu: der heilige Stanislaus Kostka, Jesuitennovize in Rom, der heilige Johannes Kantius, Professor an der Jagiellonischen Universität in Kraków, der heilige Andreas Bobola, Märtyrer in Ostpolen, 1938 heiliggesprochen, und andere Heilige bis hin zum Franziskanerpater Maximilian Kolbe, dem Märtyrer vom Konzentrationslager Auschwitz, der sein Leben für seine Mitbrüder freiwillig hingab. Gegenwärtig warten in Rom etwa 30 polnische Kandidaten auf ihre Heilig- und Seligsprechung. Unser Volk ehrt seine Heiligen und betrachtet sie als die edelste Frucht, die ein christliches Land hervorbringen kann.

Die obengenannte polnische Universität in Kraków war neben Prag die erste dieser Art im ganzen osteuropäischen Raum. Gegründet schon im Jahre 1363 von König Kasimir dem Großen (Kazimierz Wielki), war sie Jahrhunderte hindurch Zentrum nicht nur politischer, sondern auch universaler europäischer Kulturstrahlung nach allen Richtungen, im besten Sinne des Wortes. Im 15. und 16. Jahrhundert, als die schlesischen Piastenländer nicht mehr zum polnischen Königreich gehörten, studierten in Kraków und dozierten daselbst Tausende von Studenten und Professoren aus Wratislavia (Breslau), Raciborz (Ratibor), Gliwice (Gleiwitz), Glogów (Glogau), Nyse (Neisse), Opole (Oppeln) und vielen anderen Städten Schlesiens. Ihre Namen und die Namen ihrer Geburtsorte sind in diesem polnischlateinischen Idiom in den alten Universitätsregistern verzeichnet. Auch Nicolaus Kopernik (Copernicus) wird da namentlich angeführt. Er studierte in Kraków Astronomie bei Professor Martin Bylica. Hunderte von Gelehrten von höchstem wissenschaftlichen Rang hat diese Universität hervorgebracht und der europäischen Kultur geschenkt: Mathematiker, Physiker, Mediziner, Rechtsgelehrte, Astronomen, Historiker und Kulturphilosophen. Unter ihnen befindet sich auch der berühmte Paulus Włodkowic, Rektor der Krakauer Universität, der auf dem Konzil in Konstanz frank und frei, mit höchster Gelehrtenautorität, eine für damalige Zeiten unerhörte religiöse und humane Toleranz lehrte und mit großem persönlichem Mut den Standpunkt vertrat: Die heidnischen Völker Osteuropas seien kein Freiwild, das man mit Feuer und Schwert bekehren soll und darf. Sie haben natürliche Menschenrechte genauso wie die Christen...

Włodkowic war sozusagen der klassische Ausdruck des polnischen toleranten und freiheitlichen Denkens. Seine Thesen waren gegen die 
deutschen Ordensritter, die sogenannten „Kreuzritter“, gerichtet, die damals im slawischen Norden und in den preußischen und baltischen Ländern die dortigen Ureinwohner eben mit Feuer und Schwert bekehrten und für das europäische Christentum und sein Symbol, das Kreuz, aber auch für die Kirche, in deren Namen sie auftraten, im Laufe der Jahrhunderte eine furchtbare und äußerst kompromittierende Belastung geworden sind. Noch heute, nach vielen Generationen und Jahrhunderten, ist die Bezeichnung „Krzyżak» (Kreuzritter) Schimpfwort und Schreckgespenst für jeden Polen und wird leider nur all zu oft von alters her mit dem Deutschtum identifiziert. Aus dem Siedlungsgebiet der „Kreuzritter“ sind später jene Preußen hervorgegangen, die alles Deutsche in polnischen Landen in allgemeinen Verruf brachten. Sie sind in der geschichtlichen Entwicklung repräsentiert durch folgende Namen: jener Albrecht von Preußen, Friedrich der sog. Große, Bismarck und schließlich Hitler als Endpunkt.

Friedrich II. wird seit jeher vom ganzen polnischen Volk als der Haupturheber der Teilung Polens angesehen, und zweifellos nicht ganz zu Unrecht. Hundertfünfzig Jahre lebte das polnische Millionenvolk aufgeteilt von den drei damaligen Großmächten: Preußen, Rußland und Österreich, bis es 1918 am Ende des Ersten Weltkrieges langsam aus seinem Grabe hervorkommen konnte; bis zum äußersten geschwächt, begann es damals wieder unter größten Schwierigkeiten eine neue eigenstaatliche Existenz...

Nach kurzer Unabhängigkeit von etwa 20 Jahren (1918 bis 1939) brach über das polnische Volk ohne seine Schuld das herein, was man euphemistisch einfach als Zweiten Weltkrieg bezeichnet, was aber für uns Polen als totale Vernichtung und Ausrottung gedacht war. Über unser armes Vaterland senkte sich eine furchtbare finstere Nacht, wie wir sie seit Generationen nicht erlebt hatten. Sie wird bei uns allgemein „,deutsche Okkupationszeit“ genannt und ist unter diesem Namen in die polnische Geschichte eingegangen. Wir waren alle macht- und wehrlos. Das Land war übersät mit Konzentrationslagern, in denen die Schlote der Krematorien Tag und Nacht rauchten. Über sechs Millionen polnischer Staatsbürger, darunter der Großteil jüdischer Herkunft, haben diese Okkupationszeit mit ihrem Leben bezahlen müssen. Die führende polnische Intelligenzschicht wurde einfach weggefegt. Zweitausend polnische Priester und fünf Bischöfe (ein Viertel des damaligen Episkopates) wurden in Lagern umgebracht. Hunderte von Priestern und Zehntausende von Zivilpersonen wurden bei Ausbruch des Krieges an Ort und Stelle erschossen (778 Priester allein in der Diözese Kulm). Die Diözese Włocławek 
allein verlor im Kriege 48 Prozent ihrer Priester, die Diözese Kulm 47 Prozent. Viele andere waren ausgesiedelt. Alle mittleren und höheren Schulen waren geschlossen. Die Priesterseminarien waren aufgehoben. Jede deutsche Uniform, nicht nur die SS, wurde für alle Polen nicht nur ein Schreckgespenst, sondern auch Gegenstand des Deutschenhasses. Alle polnischen Familien hatten ihre Todesopfer zu beklagen. Wir wollen nicht alles aufzählen, um die noch nicht vernarbten Wunden nicht wieder aufzureißen. Wenn wir an diese polnische, furchtbare Nacht erinnern, dann nur deswegen, damit man uns heute einigermaßen versteht, uns selbst und unsere heutige Denkart... Wir versuchen zu vergessen. Wir hoffen, daß die Zeit der große göttliche Kairos die geistigen Wunden langsam heilen wird.

Nach alledem, was in der Vergangenheit geschehen ist leider erst in der allerneuesten Vergangenheit, ist es nicht $\mathrm{zu}$ verwundern, daß das ganze polnische Volk unter dem schweren Druck eines elementaren Sicherheitsbedürfnisses steht und seinen nächsten Nachbarn im Westen immer noch mit Mißtrauen betrachtet. Diese geistige Haltung ist sozusagen unser Generationenproblem, das, Gott gebe es, bei gutem Willen schwinden wird und schwinden muß. In den schweren politischen und geistigen Nöten des Volkes, in seiner jahrhundertelangen Zerrissenheit sind die katholische Kirche und die Heilige Jungfrau immer der Rettungsanker und das Symbol der nationalen Einheit des Volkes geblieben, zusammen mit der polnischen Familie. In allen Freiheitskämpfen während der Unterdrückungszeit gingen die Polen mit diesen Symbolen auf die Barrikaden, die weißen Adler auf der einen Seite die Muttergottes auf der anderen Seite der Freiheitsfahne. Die Devise war immer: „Für eure und unsere Freiheit.“

Das ist etwa in ganz allgemeinen Abrissen gezeichnet die tausendjährige Entwicklung der polnischen Kulturgeschichte mit besonderer Berücksichtigung der deutschpolnischen Nachbarschaft. Die Belastung der beiderseitigen Verhältnisse ist immer noch groß und wird vermehrt durch das sogenannte „heiße Eisen“ dieser Nachbarschaft. Die polnische Westgrenze an Oder und Neiße ist, wie wir wohl verstehen, für Deutschland eine äußerst bittere Frucht des letzten Massenvernichtungskrieges zusammen mit dem Leid der Millionen von Flüchtlingen und vertriebenen Deutschen (auf interalliierten Befehl der Siegermächte Potsdam 1945! geschehen). Ein groBer Teil der Bevölkerung hatte diese Gebiete aus Furcht vor der russischen Front verlassen und war nach dem Westen geflüchtet. Für unser Vaterland, das aus dem Massenmorden nicht als Siegerstaat, sondern bis zum äußers- 
ten geschwächt hervorging, ist es eine Existenzfrage (keine Frage „größeren Lebensraumes"!); es sei denn, daß man ein über 30MillionenVolk in den engen Korridor eines „Generalgouvernements“ von 1939 bis 1945 hineinpressen wollte ohne Westgebiete; aber auch ohne Ostgebiete, aus denen seit 1945 Millionen von polnischen Menschen in die „Potsdamer Westgebiete“ hinüberströmen mußten. Wo sollten sie auch damals hin, da ja das sogenannte Generalgouvernement zusammen mit der Hauptstadt Warschau in Schutt und Trümmern lag. Die Vernichtungswellen des letzten Krieges sind nicht nur einmal, wie in Deutschland, sondern seit 1914 mehrere Male über die polnischen Lande hinweggebraust, und zwar hin und zurück wie apokalyptische Reiter, und haben jedesmal Schutt und Trümmer, Armut, Krankheit, Seuchen, Tränen, Tod sowie wachsende Vergeltungs und Haßkomplexe hinterlassen.

Seid uns wegen dieser Aufzählung dessen, was im letzten Abschnitt unserer tausend Jahre geschehen ist, liebe deutsche Brüder, nicht gram! Es soll weniger eine Anklage als vielmehr eine eigene Rechtfertigung sein! Wir wissen sehr wohl, wie ganz große Teile der deutschen Bevölkerung jahrelang unter übermenschlichem nationalsozialistischem Gewissensdruck standen, wir kennen die furchtbaren inneren Nöte, denen seinerzeit rechtschaffene und verantwortungsvolle deutsche Bischöfe ausgesetzt waren, um nur die Namen Kardinal von Faulhaber, von Galen, von Preysing zu erwähnen. Wir wissen um die Märtyrer der weißen Rose, die Widerstandskämpfer des 20. Juli, wir wissen, daß viele Laien und Priester ihr Leben opferten (Lichtenberg, Metzger, Klausener und viele andere). Tausende von Deutschen teilten als Christen und Kommunisten in den Konzentrationslagern das Los unserer polnischen Brüder...

Und trotz alledem, trotz dieser fast hoffnungslos mit Vergangenheit belasteten Lage, gerade aus dieser Lage heraus, hochwürdige Brüder, rufen wir Ihnen zu: Versuchen wir zu vergessen! Keine Polemik, kein weiterer kalter Krieg, aber der Anfang eines Dialogs, wie er heute vom Konzil und von Papst Paul V1. überall angestrebt wird. Wenn echter guter Wille beiderseits besteht und das ist wohl nicht zu bezweifeln, dann muß ja ein ernster Dialog gelingen und mit der Zeit gute Früchte bringen trotz allem, trotz heißer Eisen. Es scheint uns gerade im ökumenischen Konzil ein Gebot der Stunde zu sein, daß wir diesen Dialog auf bischöflicher Hirtenebene beginnen, und zwar ohne Zögern, daß wir einander näher kennenlernen, unsere gegenseitigen Volksbräuche, den religiösen Kult und Lebensstil, in 
242 Volume 3 (2013) Number 2, p. 233-244

der Vergangenheit verwurzelt und gerade durch diese Kulturvergangenheit bedingt.

Wir haben versucht, uns mit dem gesamten polnischen Gottesvolk auf die Tausendjahrfeier durch die sogenannte große Novene unter dem hohen Patronat der allerseligsten Jungfrau Maria vorzubereiten. Neun Jahre hindurch (1957 bis 1965) haben wir im Sinne des ,per Mariam ad Jesum“ die Kanzel in Polen, aber auch die gesamte Seelsorge auf wichtige moderne Seelsorgeprobleme und soziale Aufgaben ausgerichtet: Jugendseelsorge, sozialer Aufbau in Gerechtigkeit und Liebe, soziale Gefahren, nationale Gewissenserforschung, Ehe- und Familienleben, katechetische Aufgaben und ähnliche.

Das ganze gläubige Volk nahm auch geistig regsamsten Anteil am Ökumenischen Konzil durch Gebet, Opfer und Bußwerke. Während der Konzilssitzungen fanden jeweils in allen Pfarrgemeinden Bittandachten statt, und das heilige Bild der Muttergottes sowie die Beichtstühle und Kommunionbänke in Częstochowa waren wochenlang belagert von Pfarrdelegationen aus ganz Polen, die durch persönliches Opfer und Gebet helfen wollten.

Schließlich haben wir uns in diesem Jahr, dem letzten der großen Novene, alle der Mutter Gottes geweiht, Bischöfe, Priester, Ordensleute sowie alle Stände unseres gläubigen Volkes. Vor den ungeheuren Gefahren moralischer und sozialer Art, welche die Seele unseres Volkes, aber auch seine biologische Existenz bedrohen, kann uns nur die Hilfe und Gnade unseres Erlösers retten, die wir durch die Vermittlung seiner Mutter, der allerseligsten Jungfrau, herabflehen wollen. Voll kindlichen Vertrauens werfen wir uns in ihre Arme. Nur so können wir innerlich frei werden als dienende und zugleich freie Kinder ja sogar als „Sklaven Gottes“, wie es der heilige Paulus nennt.

Wir bitten Sie, katholische Hirten des deutschen Volkes, versuchen Sie auf Ihre eigene Art und Weise, unser christliches Millenium mitzufeiern, sei es durch Gebet, sei es durch einen besonderen Gedenktag. Für jede Geste dieser Art werden wir Ihnen dankbar sein. Überbringen Sie auch, wir bitten Sie darum, unsere Grüße und unseren Dank den deutschen evangelischen Brüdern, die sich mit uns und mit Ihnen abmühen, Lösungen für unsere Schwierigkeiten zu finden.

In diesem allerchristlichsten und zugleich sehr menschlichen Geist strecken wir Ihnen unsere Hände hin in den Bänken des zu Ende gehenden Konzils, gewähren Vergebung und bitten um Vergebung. Und wenn Sie, 
deutsche Bischöfe und Konzilsväter, unsere ausgestreckten Hände brüderlich erfassen, dann erst können wir wohl mit ruhigem Gewissen in Polen auf ganz christliche Art unser Millennium feiern. Wir laden Sie dazu herzlichst nach Polen ein.

Das walte der barmherzige Erlöser und die Jungfrau Maria, die Königin Polens, die Regina Mundi und Mater Ecclesiae.

Rom, 18. November 1965

Die Unterzeichner des polnischen Briefes:

Stefanus Cardinalis Wyszyński, Primas Poloniae

Antonius Baraniak, Archiepiscopus Posnaniensis

Boleslaus Kominek, Archiepiscopus Tit. in Wrocław

Carolus Wojtyła, Archiepiscopus Metropolita Cracoviensis

Antonius Pawłowski, Episcopus Vladislaviensis

Casimirus Joseph Kowalski, Episcopus Culmensis

Michael Klepacz, Episcopus Lodzensis,

Czeslaus Falkowski, Episcopus Lomzensis

Petrus Katwa, Episcopus Lublinensis

Franciscus Jop, Episcopus in Opole

Herbertus Bednorz, Episcopus Coadiutor Katovicensis

Stefanus Bareła, Episcopus Czestochoviensis

Bogdanus Sikorski, Episcopus Plocensis

Edmundus Nowicki, Episcopus Gedanensis

Joannes Jaroszewicz, Admin. Apost. Kielcensis

Georgius Ablewicz, Episcopus Tarnoviensis

Joseph Drzazga, Episcopus Vic. co. p. Olsztyn

Stanislaus Jakiel, Vic. Cap. Przemyśl

Andreas Wronka, Episcopus Auxil. in Wroclaw

Venceslaus Majewski, Episcopus Auxil. Varsaviensis

Georgius Stroba, Episcopus Auxil. in Gorzów

Franciscus Jedwabski, Episcopus Auxil. in Poznań

Julianus Groblicki, Episcopus Auxil. Cracoviensis

Carolus Pękala, Episcopus Auxil. in Tarnów

Zygfrydus Kowalski, Episcopus Auxil. Culmensis

Georgius Modzelewski, Episcopus Auxil. Varsaviensis

Ioannes Wosiński, Episcopus Auxil. Plocensis

Bogdanus Bejze, Episcopus Auxil. Lodzensis 
244 Volume 3 (2013) Number 2, p. 233-244

Thaddaeus Szwagrzyk, Episcopus Auxil. Czestochoviensis Venceslaus Skomorucha, Episcopus Auxil. in Siedlce Ioannes Zaręba, Episcopus Auxil. Vladislaviensis Henricus Grzondziel, Episcopus Auxil. in Opole Josephus Kurpas, Episcopus Auxil. Katovicensis Ladislaus Rubin, Episcopus Auxil. Gnesnensis Paulus Latusek, Episcopus Auxil. in Wroclaw Joannes Czerniak, Episcopus Auxil. in Gniezno. 